\title{
Inteligencia emocional: una competencia para fortalecer el desarrollo cognitivo, el pensamiento crítico, la toma de decisiones y el rendimiento académico
}

\section{Emotional intelligence: a competence to strengthen cognitive development, critical thinking, the taking decisions and academic performance}

Julio César Freire Pazmiño. ${ }^{1}$, Blanca Maritza Guzmán Núñez. ${ }^{2}$, Julio Rafael Cabrera López. ${ }^{3}$ \& Mónica Narciza López Pazmiño. ${ }^{4}$

Recibido: 10-02-2019 / Revisado: 15-02-209 /Aceptado: 04-03-2019/ Publicado: 05-04-2019

\begin{abstract}
.
DOI: https://doi.org/10.33262/cienciadigital.v3i2.472

In Ecuador, advances in the education system are enhanced by the use and management of Curriculum Update and Strengthening, a document issued by the Ministry of Education; for this reason, the study conducted with the theme "Emotional intelligence: a competence to strengthen cognitive development, critical thinking, decision-making and academic performance" determine the causes that impair school performance with sustenance in pedagogy constructivist from an integral formation; in the research qualitative and quantitative approaches were used, while the modality was field, bibliographic and documentary, in the types and levels the exploratory, descriptive, explanatory and correlational excelled; consolidated work with a population of ninetyseven people under study: eighty-five students of basic general education and twelve teachers of the Rumiñahui Educational Unit; the data collection was developed through the argumentative and comprehensive perspective; the technique the survey with its instrument the structured questionnaire facilitated the processing of the information through the criteria of validity and reliability that contributed to the verification of the hypothesis and determination of conclusions obtaining as a result that the emotional

${ }^{1}$ Universidad Tecnológica Indoamérica, Facultad de Ciencias Humanas, de la Educación y Desarrollo Social, Carrera de Educación Básica e Inicial. Ambato, Ecuador. juliofreire@uti.edu.ec

${ }^{2}$ Universidad Técnica de Ambato. Facultad de Ciencias Humanas y de la Educación. Carrera de Psicología Educativa. Ambato, Ecuador. blancaguzmán@uta.edu.ec

${ }^{3}$ Universidad Tecnológica Indoamérica, Facultad de Ciencias Humanas, de la Educación y Desarrollo Social, Carrera de Educación Básica e Inicial. Ambato, Ecuador. juliocabrera@uti.edu.ec

${ }^{4}$ Universidad Tecnológica Indoamérica, Facultad de Ciencias Humanas, de la Educación y Desarrollo Social, Carrera de Educación Básica e Inicial. Ambato, Ecuador. monicalopez@uti.edu.ec
\end{abstract}


competences improve the active participation, the practice of values, autonomy, selfcontrol and self-confidence, contributing in teaching with quality, efficiency and productivity.

Keywords: competence, training, intelligence, participation, performance.

\section{Resumen.}

En el Ecuador, los avances en el sistema educativo toman realce con la utilización y manejo de la Actualización y Fortalecimiento Curricular, documento emitido por el Ministerio de Educación; por esta razón, el estudio efectuado con la temática "La inteligencia emocional: una competencia para fortalecer el desarrollo cognitivo, el pensamiento crítico, la toma de decisiones y el rendimiento académico", determina las causas que perjudican en el desempeño escolar con sustento en la pedagogía constructivista desde una formación integral; en la investigación se utilizó los enfoques cualitativo y cuantitativo, mientras la modalidad fue de campo, bibliográfica y documental, en los tipos y niveles sobresalió el exploratorio, descriptivo, explicativo y correlacional; trabajo afianzado con una población de noventa y siete personas objeto de estudio: ochenta y cinco estudiantes de educación general básica y doce docentes de la Unidad Educativa Rumiñahui; la recolección de datos se desarrolló a través de la perspectiva argumentativa y comprensiva, la técnica la encuesta con su instrumento el cuestionario estructurado facilitó el procesamiento de la información mediante los criterios de validez y fiabilidad que contribuyeron a la comprobación de la hipótesis y determinación de conclusiones obteniendo como resultado que las competencias emocionales mejoran la participación activa, la práctica de valores, la autonomía, el autocontrol y autoconfianza, aportando en la enseñanza con calidad, eficiencia y productividad.

Palabras claves: Competencia, Formación, Inteligencia, Participación, Rendimiento.

\section{Introducción}

\section{Educación en el proceso enseñanza aprendizaje y el desarrollo de nuevas competencias}

El sistema educativo ecuatoriano; se encuentra orientado por el Ministerio de Educación, que tiene por objeto potenciar el aprendizaje infantil, promover el bienestar en los diferentes entornos a través de experiencias significativas, contribuir en la inclusión e interculturalidad, el respeto y la convivencia; desde esta perspectiva autores como (Caicedo, Velasco, \& Valdés, 2018) exponen que la realización de actividades en el aula promueven la inteligencia emocional, el desarrollo cognitivo, las competencias intelectuales y solución de problemas con responsabilidad; cultiva actitudes, conductas, pensamientos y juicios críticos que favorecen en la autonomía, motivación e identidad personal. 
Desde distintas teorías psicológicas (Necuzzi, 2018) señala: la inteligencia emocional abre la posibilidad de exteriorizar sensaciones, pensamientos y emociones (Camilloni, 2016) que refuerzan el desarrollo cognoscitivo, las funciones básicas mentales y participación activa en el aprendizaje, debido a la diversidad de experiencias adquiridas que benefician en la resolución de conflictos (Aguirre, Vazquez, \& Bazan, 2019) pasando de un desequilibrio al equilibrio. Es decir, el docente a través de diferentes estrategias mejora el pensamiento reflexivo, la intuición y compromiso, en una perspectiva autónoma, cognitiva y social.

\section{La inteligencia en el desarrollo cognitivo y emocional}

El desarrollo cognitivo incluye las funciones básicas, reorganiza los procesos mentales, fortalece las conductas basándose en la experiencia, conduce las emociones, afianza la personalidad, la comunicación de experiencias, expresión de sentimientos, manejo de reacciones, aceptación, auto control, capacidad para resolver dificultades; mientras, el aprendizaje es un proceso que guía y orienta en la enseñanza direccionando las nuevas competencias, transformando la metodología del docente, siendo el maestro el orientador en el saber del educando; es así como (Imaculada, 2016) menciona, a medida que transcurre el tiempo la educación ha enfrentado cambios en los aspectos pedagógico, didáctico y metodológico, conllevando a mejorar la práctica formativa, las relaciones personales e interpersonales, la socialización y convivencia.

La comunicación entre el educando y el educador en el ambiente áulico es un elemento esencial en el proceso de enseñanza, el intercambio de sapiencias y toma de decisiones; (Beatriz, 2015) el diálogo como forma de información aporta a la transmisión, construcción del conocimiento y formación del individuo autónomo e independiente con capacidad de enfrentar los problemas en el diario vivir; por lo expuesto, la interacción establecida en el aula o salón de clase permite implementar nuevas metodologías, métodos y estrategias con el objetivo de despertar la atención de los estudiantes para lograr el aprendizaje significativo y colaborativo; es decir el docente debe innovar sus conocimientos transformando las clases tradicionales (repetición de contenidos y memorización).

Para (Buitrago \& Herrera, 2014) en el ambiente educativo es necesario estimular la participación de los estudiantes a través del diálogo mediante la formulación de preguntas que permitirán la aportación de ideas concretas, pensamientos lógicos, despertando la atención y el interés de los educandos en el aprendizaje de contenidos, la práctica de valores, el desenvolvimiento personal, habilidad en el trato con los demás, conocimiento de sí mismo, autocontrol de impulsos, manejo de conflictos y solución de desacuerdos.

(Jaramillo, 2016) el proceso de enseñar y aprender requiere de paciencia, sobretodo en áreas de complejidad que precisa del razonamiento lógico, argumentación fundamentada y 
memorización de contenidos con base científica; elementos que pueden afectar o favorecer en la educación; (González, 2017) aporta en la actividad cognoscitiva del ser humano, beneficia en los saberes, destrezas, costumbres y formación en un pensamiento científico mediante la investigación, el conocimiento, la dialéctica; es por ello que el docente tiene como función en la enseñanza y aprendizaje establecer una relación lógica y reflexiva entre educador y educando; (Aparicio, 2014) en el ambiente escolar impulsa, agiliza, encamina, reconoce y evalúa el discernimiento aplicando métodos apropiados; relacionando el aspecto conceptual con la adquisición de nueva información.

\section{Inteligencia emocional en el desarrollo cognitivo, el pensamiento y rendimiento.}

La formación escolar del ser humano incluye conocimientos, competencias, facultades y potencialidades, componentes que conllevan a la autonomía, autoestima, autocontrol en la exteriorización de pensamientos; desde un enfoque psicopedagógico analiza la realidad educativa con el propósito de detectar dificultades y atender a las necesidades educativas en un margen de respeto, responsabilidad, equidad y compromiso.

Para (Gutiérrez, 2018) el pensamiento crítico es un elemento que beneficia en la habilidad expresiva, las destrezas cognitivas, incluye la interpretación, evaluación, inferencia, explicación y auto regulación; según (Barnett, 2015) el docente es el orientador, motiva al descubrimiento e investigación; (Standish \& Thoilliez, 2018) contribuye en el desarrollo de nuevas actitudes y formas de pensar que consolidan la apropiación del saber con especial énfasis en la dimensión emocional siendo el educando protagonista activo de sus reflexiones, análisis, aplicación de valores de responsabilidad, equidad, respeto e inclusión vinculando la teoría y experiencia en la solución de problemas.

El rendimiento académico incluye los componentes del desarrollo cognitivo, procedimental, actitudinal, sustentándose en la pedagogía intuitiva, el construccionismo e inteligencia emocional, incentiva en la adquisición de juicios valorativos, expresiones concretas y criterios significativos, según lo mencionado, los maestros deben incorporar en la enseñanza nuevas estrategias que permiten mejorar las habilidades para alcanzar el aprendizaje significativo; (Calderon, 2017) en su artículo señala los factores que pueden afectar de forma directa en el pensamiento al generar dificultades en la comprensión de conocimientos impartidos por los docentes, insuficiente razonamiento; (López Pamiño, 2017), el desempeño de los estudiantes está relacionado con la motivación del docente, la planificación de contenidos curriculares, el plan de clase, los recursos y materiales, aspectos que han relegado la empatía, el autoconocimiento de emociones y sentimientos.

Para (Rojas, 2017) los estados de ánimo, conducta inapropiada y comportamientos inadecuados afectan el rendimiento académico, provocando estrés, agotamiento físico, psicológico y mental; (López, Sagñay, Vega, \& Mera, 2019) factores que obstaculizan la 
estimulación del área cognitiva; incrementa cambios conductuales que repercuten en el progreso del aprendizaje, exteriorizando miedo, nerviosismo, desesperación en el cumplimiento de tareas y lecciones; (Borda, 2013) manifiesta, dentro del desempeño escolar la motivación es el centro productor de energía que impulsa el estudio y realización del ser humano despertando el interés por el aprender, aportando las capacidades cognitivas, la resolución de conflictos, las habilidades sociales y comunicativas con impacto en la convivencia escolar.

\section{Metodologia}

El estudio efectuado se sustenta en el derecho del ser humano a una educación de calidad en igualdad de oportunidades, por lo expuesto, en la metodología de la investigación se aplicó un enfoque cualitativo que considera en las dimensiones la inteligencia emocional, el desarrollo cognitivo, el pensamiento crítico, la toma de decisiones y el rendimiento académico; desde el planteamiento del problema, la conceptualización, operacionalización, recolección e interpretación de datos prevaleció el estudio cuantitativo al tener como propósito el análisis estadístico que benefició en la comprobación de la hipótesis y el establecimiento de resultados.

En la modalidad básica el estudio de campo favoreció en la recolección de información directa mediante la encuesta; mientras el modo bibliográfico - documental, permitió profundizar en el conocimiento de la inteligencia emocional, el desarrollo cognitivo, el pensamiento crítico, la toma de decisiones y el rendimiento académico, empleándose artículos científicos y libros de diferentes autores.

La indagación exploratoria se encauzó en el análisis de la inteligencia emocional, el desarrollo cognitivo, el pensamiento crítico, la toma de decisiones y el rendimiento académico siendo un tema de escaso estudio en el proceso educativo; su aplicación facilitó la formulación del problema, el planteamiento de la hipótesis, el establecimiento de conclusiones y resultados.

La indagación de tipo descriptivo en el método científico impulsó la observación y definición de las causas que influyen en el desarrollo de la inteligencia emocional; no obstante la investigación correlacional permitió establecer el grado de relación entre las variables para efectuar futuras investigaciones en diferentes espacios educativos.

La investigación para efectos del estudio a través del análisis argumentativo y estadístico se organizó en una totalidad de 85 educandos de décimo año de educación general básica, distribuido en los paralelos " $\mathrm{A}$ " $\mathrm{y}$ " $\mathrm{B}$ " junto con 12 docentes que desarrollan su labor en la Unidad Educativa Rumiñahui, de la ciudad de Ambato; detallándose a continuación: 


\section{Resultados.}

Tabla $\mathbf{N}^{\mathbf{0}}$ 1. Población

\begin{tabular}{lll}
\hline POBLACIÓN & FRECUENCIA & PORCENTAJE \\
\hline Estudiantes Décimo año “A” & 40 & $41 \%$ \\
Estudiantes Décimo año "B" & 45 & $47 \%$ \\
Docentes & 12 & $4 \%$ \\
Total & 97 & $100 \%$ \\
\hline
\end{tabular}

Fuente: Secretaría de la Institución

\section{Elaborado por: Guzmán Núñez Blanca Maritza}

La muestra estuvo conformada por el total de la población, desde un matiz no probabilístico que presenta la profundización en la problemática en el conocimiento, la comprensión, el entendimiento y reflexión; (López Pazmiño, 2016) componentes que apoyan en la determinación de respuestas a ítems planteados en el estudio mediante el juicio valorativo de la investigadora (López, 2016) señala: en poblaciones pequeñas es importante tomar la muestra en su totalidad; es decir, se requiere de un número mayor a 100 individuos para efectuar un cálculo muestral; por las razones expuestas se aplicó los instrumentos de recopilación de información a 97 personas objeto de análisis.

La información cuantitativa facilitó el análisis estadístico mediante la utilización de programas informáticos, empleándose en la redacción de ítems un lenguaje concreto y comprensible que disminuye el nivel de complejidad de datos. El cuestionario se integró con un total de 13 interrogantes incluyendo alternativas cerradas de aspectos numéricos.

La operacionalización de la variable independiente: inteligencia emocional se presenta con su conceptualización, con sus dimensiones e indicadores autoconciencia (emociones autoconfianza), motivación (perseverancia e iniciativa), destrezas sociales (toma de decisiones y liderazgo), empleándose seis ítems básicos.

La operacionalización de la variable independiente rendimiento académico incluye la definición con las dimensiones e indicadores capacidades cognitivas (razonamiento pensamiento crítico, argumentación), aprendizaje (comprensión, autonomía, retención) evaluación (formativa y progresiva) empleándose siete ítems básicos; en la técnica se empleó la encuesta y el instrumento el cuestionario estructurado dirigido a docentes y estudiantes con el propósito de encontrar una alternativa de solución al problema detectado. 
Tabla $\mathbf{N}^{\mathbf{0}}$ 2. Ítems aplicados a estudiantes

\begin{tabular}{cl}
\hline №. & \multicolumn{1}{c}{ Preguntas } \\
\hline 1 & ¿Reconoce las emociones propias y las conductas de los demás? \\
2 & ¿Demuestra autoconfianza y seguridad en su desempeño académico? \\
3 & ¿Es perseverante en el cumplimiento de metas? \\
4 & ¿Manifiesta iniciativa, responsabilidad y compromiso en el cumplimiento de \\
tareas escolares y familiares? \\
5 & ¿Posee capacidad para manejar conflictos, tomar decisiones y resolver \\
6 & $\begin{array}{l}\text { ¿Desacuerdos? } \\
7\end{array}$ \\
8 & ¿Organiza la información y comunica sus ideas con claridad y precisión? \\
9 & ¿Asimila conocimientos para el desarrollo de nuevas experiencias? \\
10 & ¿Usted comprende, argumenta, analiza durante el proceso enseñanza \\
& aprendizaje? \\
11 & ¿Usted fortalece la integración, demuestra autonomía e independencia? \\
12 & ¿La información presentada por el maestro impulsa la curiosidad, aumenta la \\
& retención y entendimiento?
\end{tabular}

Fuente: Guía de preguntas aplicada en la investigación

Elaborado por: Grupo de investigadores

Por ser un estudio descriptivo, explicativo y correlacional con enfoque cualitativo y cuantitativo, en la comprobación de la hipótesis se establece una combinación de frecuencias de la variable independiente inteligencia emocional y como variable dependiente rendimiento académico, incluyendo como dimensiones: reconocimiento de emociones, autoconfianza, seguridad, manejo de conflictos, toma de decisiones, liderazgo, pensamiento crítico, integración, autonomía e independencia.

Mediante la utilización de la muestra aleatoria se seleccionan dos interrogantes del cuestionario aplicado a los educandos (interrogante 5: manejo de conflictos, toma de decisiones); (interrogante 8: comprensión, pensamiento crítico); con un nivel de significación del $0.05 \%$, una confiabilidad del $95 \%$ y 6 grados de libertad con equivalencia del 12.59 . 
Ítem N. 5: ¿Usted tiene capacidad para manejar conflictos, tomar decisiones y resolver desacuerdos?

Tabla N $\mathbf{N}^{\mathbf{2}}$ 2. Maneja de conflictos, toma de decisiones

\begin{tabular}{lll}
\hline ALTERNATIVAS & FRECUENCIA & PORCENTAJE \\
\hline $\mathrm{Si}$ & 21 & $25 \%$ \\
No & 64 & $75 \%$ \\
\hline Total & 85 & $100 \%$ \\
\hline
\end{tabular}

Fuente: Guía de preguntas aplicada en la investigación

Elaborado por: Grupo de investigadores

El 25\% si maneja conflictos; mientras el 75\% no toma de decisiones ni resuelve desacuerdos, de esta manera limita la capacidad en la solución de dificultades a través de una buena comunicación, lo que deteriora el diálogo, la seguridad, confianza, valoración y autoestima. Los estudiantes que actúan con autonomía han fortalecido su autoestima, las relaciones interpersonales, la comprensión el lenguaje y el reconocimiento de sí mismo, componentes que aportan en la mejora de la enseñanza aprendizaje, el pensamiento crítico, el desempeño académico, la construcción de la identidad, las formas de pensar, sentir, actuar y razonar; sin embargo los educandos que exteriorizan dificultades con la toma de decisiones presentan limitaciones en la transmisión de contenidos verbales generando sentimientos de inseguridad y aislamiento influyendo de manera negativa en la comunicación, motivación y adquisición de experiencias.

Ítem N. 8: ¿Usted comprende, expresa experiencias, situaciones, saberes con pensamiento crítico, emite juicios significativos?

Tabla $\mathbf{N}^{\mathbf{o}}$ 3. Comprende, expresa experiencias, con pensamiento crítico

\begin{tabular}{lll}
\hline ALTERNATIVAS & FRECUENCIA & PORCENTAJE \\
\hline $\mathrm{Si}$ & 31 & $38 \%$ \\
No & 54 & $62 \%$ \\
\hline Total & 85 & $100 \%$ \\
\hline
\end{tabular}

Fuente: Guía de preguntas aplicada en la investigación

Elaborado por: Grupo de investigadores

El 38\% de estudiantes encuestados han desarrollado la comprensión, siendo capaces de expresar experiencias propias, analizar situaciones y exteriorizar saberes demostrando autoconfianza y seguridad; sin embargo el $62 \%$ presenta retraimiento, desmotivación y desinterés en el proceso enseñanza aprendizaje, generando barreras en la percepción el 
autocontrol, el pensamiento crítico y el rendimiento, afectando al bienestar, el desarrollo cognitivo, las habilidades mentales, la autorrealización, autoestima y autonomía.

En su mayoría, no reconocen las conductas de los demás, lo que dificulta el control de impulsos, el equilibrio en la exteriorización de pensamientos y emociones; es decir es escasa la aplicación de metodologías que aporten en la inteligencia emocional, generando barreras en el desarrollo cognitivo y procedimental.

Además, las situaciones de conflicto repercuten en la adaptación académica, familiar y social, dificultan el aprendizaje, afectan en el rendimiento, el área cognitiva y aplicación de procesos, limitando la adquisición de nuevos conocimientos; por tal razón desde la perspectiva del pensamiento y la visión holística, el docente contribuye en el desarrollo intelectual, las capacidades y potencialidades.

La escasa la utilización de técnicas que beneficien en el rendimiento, el enfrentamiento a problemas, la capacidad de comprensión, comunicación y razonamiento influye en la observación, la toma de decisiones responsables y la participación activa en el proceso enseñanza - aprendizaje, condicionando la calidad del sistema educativo.

El diagrama de frecuencias es representado Mediante los indicadores evaluación progresiva recopilación, comprensión y monitoreo

Gráfico $\mathbf{N}^{0}$. 1. Histograma

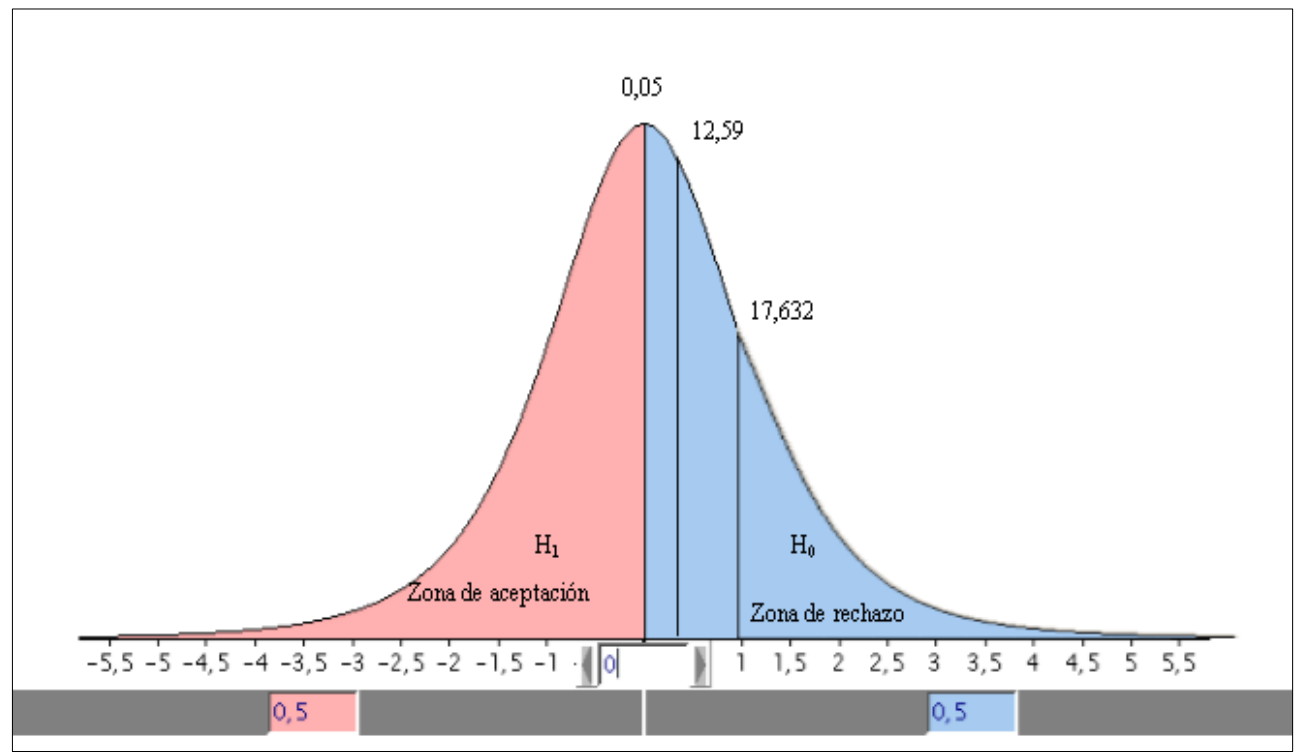

Elaborado por: Grupo de investigadores

En el histograma de distribución normal, se observa la curva asintótica con una probabilidad de 82,368 que la inteligencia emocional incide en el rendimiento académico de los estudiantes con un valor de distribución de 12,59, inferior a 17,623 es decir, la curva de 
probabilidad se encuentra sobre la mediana, (manejo de conflictos, toma de decisiones, comprensión y pensamiento crítico).

De acuerdo al resultado numérico obtenido se determina que los docentes en el ambiente educativo mediante la utilización de actividades escolares y extraescolares fortalecen la comprensión, disminuyen los conflictos en la convivencia, mejoran las relaciones familiares e integración de esta manera estimula la comunicación, los aspectos socio emocionales, el bienestar personal y escolar, influyendo en el coeficiente intelectual, las conductas, el comportamiento, la exteriorización de pensamientos.

El fortalecimiento de la habilidad en la solución de problemas disminuye la frustración posibilitando la argumentación, reflexión el desarrollo emocional, la responsabilidad educativa; a través del trabajo integrador aporta en el aprendizaje colaborativo y significativo, mejora el rendimiento, la participación e interacción.

Al tomar conciencia de las limitaciones desde la perspectiva del pensamiento crítico, los maestros deben promover la autorregulación emocional, el reconocimiento de debilidades en el ámbito educativo se propone mejorar el proceso de enseñanza aprendizaje con la finalidad de contribuir en el desarrollo de las capacidades cognitivas, el rendimiento y desempeño académico, considerando que las competencias emocionales son el resultado de la interacción entre el estudiante y el contexto.

Desde una perspectiva constructivista la aplicación de estrategias en el clima escolar reduce las conductas agresivas, comportamientos inapropiados; por tal razón en el proceso educativo el fortalecimiento de la inteligencia beneficia en la capacidad intelectual, el conocimiento, el autodominio y concentración.

Los maestros a través de diferentes actividades impulsan la práctica de valores que aporta en la colaboración, construcción de habilidades intelectuales, exteriorización de criterios manteniendo el juicio crítico, de esta forma se disminuye las barreras que repercuten en el desarrollo cognitivo, la reflexión, entendimiento, concentración, lenguaje, toma de decisiones, el trabajo en equipo, la cooperación e integración.

\section{Discusión}

Discusión la educación en el país ecuatoriano en el nivel inicial, básico, bachillerato, y superior cumple con el principio de equidad al integrar a niños, jóvenes y adultas al sistema escolar esencialmente sectores vulnerables de la sociedad; (Franco, 2014) por tal razón el estudio aporta en la labor pedagógica, didáctica y metodológica de los maestros, quienes orientan en la profundización del conocimiento y autoconocimiento (Navas, Benalcázar, López, \& Acosta, 2019) para mejorar la participación disminuyendo las barreras en el desarrollo de las capacidades físicas, mentales y psicoactivas; es decir, desde la perspectiva 
sistemática educativa el término inteligencia emocional es de importancia en el control y modificación de emociones, las habilidades cognitivas, el coeficiente intelectual y el proceso en operaciones intelectuales, por lo expuesto, la misión de la escuela es contribuir en el aprendizaje, sin embargo, la escasa aplicación de actividades que aporten en el manejo de conflictos perjudica en la toma de decisiones.

Para los investigadores (García, Hernández, \& Peláez, 2018), la ausencia de habilidades de pensamiento y capacidades intelectuales incrementa las dificultades de aprendizaje (López E. , 2005), por otro lado, repercute en el nivel cognitivo, el juicio crítico, las destrezas lingüísticas, la cooperación y solución de problemas; ante lo expuesto (López \& Acuña, 2018) señalan, los modelos educativos estimulan las competencias emocionales, autonomía intelectual, la interacción, reflexión y potenciales meta cognitivos. La labor de los docentes influye en la autorregulación de emociones, el autocontrol, la concienciación, adaptabilidad, motivación e iniciativa.

Para (Caicedo, Velasco, \& Valdés, 2018) el refuerzo de las estructuras cognitivas mejora las habilidades cognoscitivas que engloban la atención, codificación, almacenamiento y recuperación de información; no obstante (Soto, 2018) señala, la inteligencia cognoscente beneficia en la gestión de emociones propias y ajenas; entendiéndose la capacidad emocional como el conjunto de competencias qué favorecen en la intuición, regulación, valoración y percepción.

El insuficiente desarrollo de habilidades intelectuales influye en la toma de decisiones con pensamiento crítico; según (Necuzzi, 2018) en el estudio con la temática educación, enseñanza y didáctica expone la necesidad de aplicar metodologías que mejoren el diálogo, la reflexión, el control de emociones y autoestima; mientras (Camilloni, 2016) afirma que el rendimiento y desempeño son elementos que favorecen en las destrezas cognitivas; por lo expuesto, en el ambiente de aula se requiere la selección de herramientas que apoyen en las experiencias de aprendizaje; debiéndose en algunos casos reelaborar los contenidos para avanzar en el cumplimiento de objetivos educativos.

Para (Serrano, 2018) la enseñanza al definirse desde la perspectiva moral y el punto de vista etimológico se encamina al conocimiento, la resolución de situaciones problemáticas y la cooperación; a partir del criterio expuesto (Aguirre, Vazquez, \& Bazan, 2019) agrega que la empatía, autoestima y autocontrol se constituyen en elementos esenciales que facilitan la aplicación del modelo didáctico, las estrategias metodológicas, innovación en técnicas y procedimientos, de esta forma se aumenta los estándares de aprendizaje.

Según (Navas, Benalcázar, López, \& Acosta, 2019), las actividades efectuadas por los maestros refuerzan la de oportunidades educativas (González, 2017) siendo necesario cumplir con procedimientos pedagógicos que mejoran las relaciones interpersonales, 
cooperativas participativas evidenciando en los educandos el control de emociones y la toma de decisiones.

Los educandos al exteriorizar desinterés en el aprendizaje e incumplimiento de tareas, obtienen un bajo rendimiento académico (Portellano, 2014) siendo trascendental reforzar la inteligencia emocional que contribuye en la asimilación, el razonamiento y solución de problemas; (Portellano, 2014) además promueve la comunicación, el razonamiento, la comprensión e independencia en la realización de actividades escolares.

\section{Conclusiones}

- El estudio de la inteligencia emocional, el pensamiento crítico y las competencias al incidir en el rendimiento académico favorecen en la comprensión, el conocimiento sobre la información, resolución de problemas y mejora la calidad de vida en el entorno educativo, familiar y social.

- Es necesario fortalecer las características de la inteligencia emocional autoconciencia, empatía, autorregulación adaptabilidad, iniciativa, destrezas sociales, comunicación, liderazgo y trabajo en equipo, teniendo como proyección aportar en el desarrollo cognitivo y mejorar el rendimiento académico.

- La escasa realización de actividades educativas afecta en la inteligencia emocional, el desarrollo cognitivo, el pensamiento crítico, creativo e imaginativo generando barreras en la toma de decisiones y solución de conflictos en el diario vivir; por las razones mencionadas la investigación aporta en el conocimiento, la percepción y el reconocimiento de emociones, concretándose en aprendizajes significativos y duraderos.

\section{Referencias bibliográficas}

Aguirre, J., Vazquez, L., \& Bazan, S. (2019). La enseñanza del pensamiento histórico en los profesores memorables. Las buenas prácticas desde la indagación narrativa. Revista Educación. Revista Educación, 43(1), 18. Obtenido de http://www.redalyc.org:9081/home.oa?cid=1104477

Aparicio, J. J. (2014). Los estudiantes sobre el cambio conceptual y las aportaciones de la Psicologia del Aprendizaje.

Barnett, L. (2015). Motivación, tratamiento de la diversidad y rendimiento academico.

Beatriz, E. M. (2015). Influencia de la inteccion alumno - docente en el proceso de enseñanza aprendizaje. Paakat, 8.

Borda, A. (2013). Rebdimiento academico tecnicas para estudiar mejor.

Buitrago, D., \& Herrera, C. (2014). La inteligencia emocional y el tratamiento de las conductas disruptivas en el aula de clase. Ibagué, Colombia: Universidad del Tolima. 
Caicedo, L., Velasco, R., \& Valdés, A. (Agosto de 2018). La comunicación y las relaciones humanas en el proceso de enseñanza aprendizaje . VARONA, Revista CientíficoMetodológica, Edición especial, 6. doi:ISSN: 1992-8238

Calderon, N. (07 de Abril de 2017). EcuRed. Obtenido de Rendimiento Académico: https://www.ecured.cu/Rendimiento_acad\%C3\%A9mico

Camilloni, A. y. (2016). El saber didáctico. Buenos Aires. Argentina, Argentina: Paidós. Obtenido de https://lacalorconsaco.files.wordpress.com/2014/08/camilloni-el-saberdidactico.pdf

Franco, L. (2014). Construcción de la democracia escolar y social desde los procesos de convivencia en las aulas. Caldas, Colombia: Universidad de Manizales.

García, D., Hernández, J., \& Peláez, A. (2018). Evaluación del programa de desarrollo cognitivo" inteligencia XXI". Revista INFAD de Psicología. International Journal of Developmental and Educational Psychology, 1(2), 11. doi:ISSN: 0214-9877

González, E. (6 de Agosto de 2017). Inteligencia emocional, estilos de aprendizaje y rendimiento académico en futuros maestros de Educación Infantil. Universidad de La Laguna, $36 . \quad$ Obtenido de https://riull.ull.es/xmlui/bitstream/handle/915/5783/Inteligencia\%20emocional,\%20 estilos $\% 20$ de $\% 20$ aprendizaje $\% 20 y \% 20$ rendimiento $\% 20$ academico $\% 20$ en $\% 20$ futur os $\% 20$ maestros $\% 20 \mathrm{de} \% 20$ Educacion $\% 20$ Infantil..pdf?sequence $=1$

Gutiérrez, M. (2018). Estilos de aprendizaje, estrategias para enseñar. Su relación con el desarrollo emocional y aprender a aprender". Universidad Autónoma de Madrid. Departamento de Didáctica y Teoría de la Educación, 31(18), 14. Obtenido de https://www.researchgate.net/profile/Mariano_Tapias/publication/322336059_Estil os_de_aprendizaje_estrategias_para_ensenar_Su_relacion_con_el_desarrollo_emoci onal_y_aprender_a_aprender/links/5ba0ccb192851ca9ed11ff56/Estilos-deaprendizaje-estrategias-para-

Harris, A., \& Bennett, N. (2016). Eficacia Escolar y Mejoramiento Escolar. Perspectivas alternativas. Londres, Inglaterra: Continuum.

Hose, C. (4 de Marzo de 2016). Juegos para enseñarles a los niños sobre las emociones. Universidad de Huelva, 12.

Imaculada, T. (2016). El proceso de enseñanza aprendizaje y la actividad investigadora en el marco del espacio Europeo de Educación superior. Univercidad de Granada, 5.

Jaramillo, E. (2016). Pedagogia, aprendizaje, inteigencia y redimiento.

Jiménez, M. (2016). Inteligencias Múltiples en el rendimiento académico infantil. Guadalajara, México: Mc Graw Hill.

López Pamiño, M. (2017). Intervención psicopedagógica en el refuerzo académico mediante técnicas y estrategias para el fortalecimiento de las funciones psicológicas superiores en las Fundaciones Don Bosco, Santa Marianita, Proyecto Salesiano y el Gobierno Autónomo Descentralizado. Ambato, Ecuador: Universidad Técnica de Ambato. 
López Pazmiño, M. (2016). Diseño del proyecto de investigación . Ambato, Tungurahua, Ecuador: Universidad Técnica de Ambato.

López, E. (3 de Diciembre de 2005). La educación emocional en la educación infantil. Revista Interuniversitaria de Formación del Profesorado, 8(3), 12.

López, G., \& Acuña, S. (Mayo de 2018). Aprendizaje cooperativo en el aula. . Inventio, la génesis de la cultura universitaria en Morelos, 7(1), 14. doi:ISSN 2448-9026

López, M., Sagñay, F., Vega, S., \& Mera, I. (29 de Marzo de 2019). El entorno familiar y el aprendizaje cognitivo. (file:///C:/Users/PC-146/Downloads/420Texto\%20de1\%20art\%C3\%ADculo-1786-4-10-20190423.pdf, Ed.) Revista Indexada El Latindex 2.0. Revista Digital, 3(2), 18. doi:ISSN 2602-8085

Mercadé, A. (20 de Febrero de 2016). Los 8 tipos de inteligencia según Howard Gardner. Editorial Académica Española, 15.

Miñaca, M., Hervas, M., \& Laprida, I. (13 de Julio de 2013). Análisis de programas relacionados con la Educación Emocional desde el modelo propuesto por Salovey \& Mayer. Universidad La Huelva, 18.

Moreno, C. (4 de Marzo de 2016). Estrategia de conducta. Pedagogía , 16.

Murillo, F. (2006). Murillo, F.J. (Coord.) (2006). Estudios sobre Eficacia Escolar en Iberoamérica. 15 buenas investigaciones. Bogotá, Colombia: Convenio Andrés Bello.

Navas, L., Benalcázar, O., López, M., \& Acosta, J. (27 de Marzo de 2019). Education inclusive in partner-affective development: a study with students who present permanent special educational needs. Ciencia Digital, 3(2), 13. doi:DOI: https://doi.org/10.33262/cienciadigital.v9i2.390

Necuzzi, C. (2018). Educación, enseñanza y didáctica en la contemporaneidad. Palermo, Buenos Aires: Cuadernos del Centro de Estudios en Diseño y Comunicación [Ensayos]. doi:ISSN 1668-0227

Pazmiño, D. (2014). Inteligencia Emocional. Buenos Aires, Argentina: Kairos.

Portellano, J. (2014). Estimular el cerebro para mejorar la actividad mental. Madrid, España: Complutense de Madrid.

Rojas, J. B. (2017). Estres academico y expresion de ira en estudienates del nivel secundario de las Instituciones Publicas del distrito de Coishco.

Serrano, J. (2018). El documental ético: recurso procedimental, evaluación y pensamiento crítico. Revista digital de educación y formación del profesorado(15), 179-236. doi:ISSN: 1697-9745

Silva, M. (2013). Habilidades emocionales en estudiantes de educación secundaria. Michoacán, México: Universidad Michoacana de San Nicolás de Hidalgo.

Soto, M. (2018). Diseño de material didáctico como apoyo pedagógico para el desarrollo cognitivos de niños en etapa preescolar. Cali, Colombia: Pontificia Universidad Javeriana.

Standish, P., \& Thoilliez, B. (Marzo de 2018). El pensamiento crítico en crisis. Una reconsideración pedagógica en tres movimientos. Ediciones Universidad de 
Salamanca. Revista Interuniversitaria, 30(2), $16 . \quad$ doi:DOI: http://dx.doi.org/10.14201/teoredu302722

Touron, J. (2014). Factores del rendimiento académico en la Universidad. San Sebastián, España: Universidad de Navarro.

Valle, A., Cabanach, R., Rodríguez, S., Núñez, J., \& González, J. (2016). Metas académicas, estrategias cognitivas y estrategias de autorregulación del estudio. Asturias, Europa: Psicothema.

\section{PARA CITAR EL ARTÍCULO INDEXADO.}

Freire J., Guzmán B., Cabrera J., \& López M. (2019). La inteligencia emocional: una estrategia para mejorar la pedagogía crítica, el rendimiento académico y la comunicación. Revista electrónica Ciencia Digital 3(2), 645-659. Recuperado desde: http://cienciadigital.org/revistacienciadigital2/index.php/CienciaDigital/article/view/472/1081

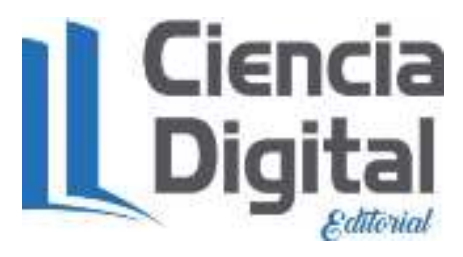

El artículo que se publica es de exclusiva responsabilidad de los autores y no necesariamente reflejan el pensamiento de la Revista Ciencia Digital.

El artículo queda en propiedad de la revista y, por tanto, su publicación parcial y/o total en otro medio tiene que ser autorizado por el director de la Revista Ciencia Digital.
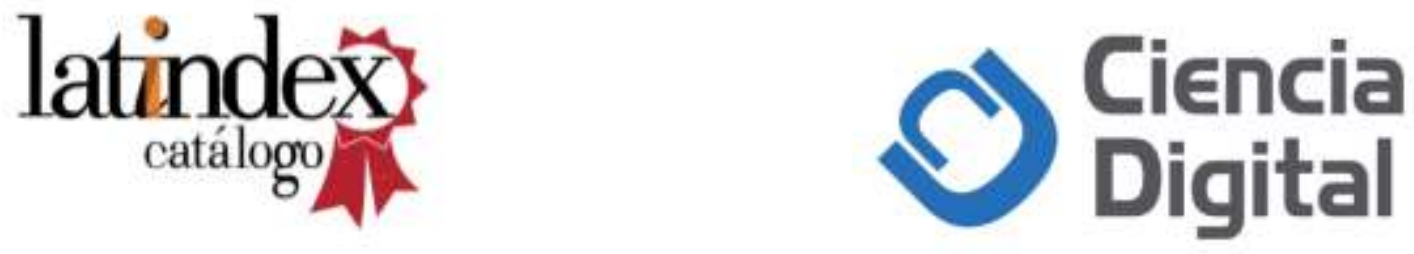